\title{
SOBRE LA POSIBILIDAD DE APLICAR LA METODOLOGÍA ORIENTADA AL PROYECTO, EN LA ENSEÑANZA DE LA INGENIERÍA DE LA UNIVERSIDAD DE TARAPACÁ-CHILE
}

\author{
Carlos Villarroel G. ${ }^{1} \quad$ Carlos Herrera S. ${ }^{2}$ \\ Recibido el 24 de noviembre 2003, aceptado el 20 de octubre de 2004
}

\begin{abstract}
RESUMEN
La metodología orientada al proyecto, incorporada a la docencia destinada a la formación de ingenieros, se presenta como una promisoria opción de enseñanza en la actualidad. Se basa en los nuevos postulados epistemológicos de la pedagogía constructivista, que concede gran importancia a la actuación del estudiante en el logro de sus aprendizajes. De esta manera, se opone al enfoque didáctico tradicional, regido por el modelo de la simple transmisión de conocimientos que imparte el profesor a sus alumnos.

El presente texto expone las razones que justifican la introducción de esta metodología a la enseñanza de la ingeniería, destacando los beneficios y ventajas que aporta su implementación en la realización del proceso de enseñanzaaprendizaje.

Además de dinamizar y activar la participación de los estudiantes, la metodología orientada al proyecto propende al logro de aprendizajes superiores, acordes con los requerimientos de la industria y de la sociedad actual.

El trabajo presenta los elementos teóricos básicos de esta opción didáctica y reflexiones en torno a la posible incorporación de ella en el sistema curricular de la Facultad de Ingeniería de la Universidad de Tarapacá, en Arica, Chile.
\end{abstract}

Palabras claves: Enseñanza de la ingeniería, metodología orientada al proyecto.

\begin{abstract}
The project-oriented methodology, incorporated in education for engineering, appears nowadays as a promising teaching option. It is based on the new epistemological postulates of the constructivistic pedagogy, that gives the student's acting a great significance in the achievement of his learnings. Thus it is opposed to the traditional didactic focusing, ruled by the simple teacher - to - student knowledge transmission model.

This paper shows the reasons that justify introduction of this methodology in engineering education, pointing out benefits and advantages provided by its implementation along the teaching-learning process.

Besides making the students participation more active and dynamic, project-oriented methodology promotes reaching higher levels of learning, according to current industry and society requirements.

This work presents the basic theoretical elements of this didactic option and reflections about its possible incorporation in the curricular system of the Engineering Faculty of Universidad de Tarapacá in Arica, Chile.
\end{abstract}

Keywords: engineering education, project-oriented teaching methodology.

\section{INTRODUCCIÓN}

Dentro del planteamiento relativo a una redefinición universitaria, el perfil del ingeniero debe adquirir una mayor trascendencia en cuanto a la problemática de su efectiva inserción en el medio industrial y en su participación en el desarrollo de tecnologías propias. Es decir, las escuelas de ingeniería y el medio industrial deberán complementarse, con el propósito de obtener un mayor y más efectivo compromiso del ingeniero, en la creación, optimización y adecuación de sistemas técnicos [1]. Al respecto, nos parece de vital importancia considerar la posibilidad de aplicar a

\footnotetext{
1 Universidad de Tarapacá. Departamento de Electrónica. Facultad de Ingeniería. 18 de septiembre 2222, Casilla 6-D, Arica-Chile, carlosvillarroel@uta.cl

${ }^{2}$ Universidad de Tarapacá. Departamento de Educación. Facultad de Educación y Humanidades. 18 de septiembre 2222, Casilla 6-D, Arica-Chile, cherrera@uta.cl
} 
nuestra enseñanza de la ingeniería, la denominada metodología orientada al proyecto, puesto que la docencia pone, de acuerdo con los principios de esta metodología, mayor énfasis en los aspectos de efectividad operacional e interpersonal, superando las prácticas de la enseñanza tradicional, destinada solamente a presentar situaciones teóricas y conceptos fundamentales. También se puede afirmar que cuando los proyectos constituyen parte importante del proceso de enseñanza-aprendizaje, se ofrece a los futuros ingenieros mayores oportunidades para desarrollar competencias que las empresas realmente requieren en la actualidad.

Una de las mayores deficiencias del modelo didáctico tradicional radica, precisamente, en que nuestros estudiantes egresan poco entrenados para enfrentar los problemas reales que se les presentarán en sus futuros empleos. Sin embargo, se espera de ellos que en la vida profesional se manifiesten bien calificados para enfrentar problemas de tipo multidisciplinarios y, por ende, capacitados para trabajar en equipos, para analizar problemas emergentes y ser capaces de involucrarse en los nuevos campos del conocimiento, relacionados con los problemas de la práctica [2]. En resumen, nuestros egresados debieran entender las conexiones que se presentan entre los diferentes y complejos problemas que se presentan en la sociedad actual [3].

Por lo tanto, la interrogante que surge en estos momentos es saber si nuestros egresados estarán preparados para enfrentar las demandas presentes $\mathrm{y}$ futuras de su profesión.

Las investigaciones realizadas al respecto, nos informan que el uso de la computación, de los idiomas extranjeros, del conocimiento práctico, de la gestión empresarial, el conocimiento y preservación del medio ambiente, la cultura y la sociedad son elementos a los cuales tanto la universidad como la industria dan un alto valor. Sin embargo, la realidad nos muestra el énfasis que la universidad suele poner en las ciencias básicas y de la ingeniería, mientras que la industria pone mayor énfasis en el uso de la informática, de los idiomas extranjeros y del conocimiento práctico. Este hecho puede explicarse por la diferencia de la naturaleza y propósitos que presentan ambos tipos de instituciones, así se produce una descoordinación entre la oferta y la demanda [1].

Las mismas investigaciones, al determinar la fuente del conocimiento profesional, revelan que después de 3 a 4 años de experiencia, la mitad de los nuevos profesionales consideran que el trabajo de proyectos constituye la fuente principal de conocimientos, mientras que un quinto de ellos es de opinión que el hecho de interactuar con los profesores constituye la principal fuente de conocimientos. Este quinto de los encuestados consideró que los cursos de educación sistemática (pregrado, postgrado, etc.) eran la fuente principal del conocimiento. En respuesta a la pregunta referida a dónde ellos aprendieron a aplicar mejor sus conocimientos, los encuestados respondieron que el trabajo de proyecto fue más importante que los cursos impartidos para lograr este propósito [1], [3].

\section{La Enseñanza Orientada por Proyectos}

El método de proyectos fue creado por W.H. Kilpatrick en 1918. Lo fundó en el análisis del pensamiento hecho por John Dewey, y su cometido fue el ensayo de una forma efectiva de enseñar. De los mismos principios que dieron origen al método de proyectos surgió el método de problemas, por obra del propio Dewey. En tanto que el método de Kilpatrick se propone actuar concretamente en el campo de la realización efectiva, el de Dewey procura desenvolverse en el campo intelectual [4].

El método de proyectos es debido, pues, a Kilpatrick, quien adhiere a la línea pragmática de Dewey. Tiene por finalidad llevar a los alumnos a realizar algo. Es un método esencialmente activo, cuyo propósito es hacer que los alumnos realicen, actúen. Es, en suma, el método de determinar una tarea y pedirle a los alumnos que le lleven a cabo.

El método de proyectos intenta imitar la vida, ya que todas las acciones del ser humano no son otra cosa que realizaciones de proyectos. El ser humano vive proyectando continuamente. Debe señalarse, empero, una diferencia entre el proyecto del adulto y el proyecto del educando. El adulto proyecta después de conoce; el educando proyecta para conocer.

El método de proyecto ha tenido amplia aplicación en la educación superior, especialmente en los países europeos y Norteamérica. Representa pedagógicamente, un paso al frente con relación al de problemas. La solución de un problema no es tan rica como la solución por la realización, la realización implica una riqueza extraordinaria de experiencia que en modo alguno consigue proporcionar la simple solución teórica [5].

El método de proyecto procura desenvolver al espíritu de iniciativa, de responsabilidad, de solidaridad y de libertad.

El proyecto es una cadena organizada de actividades, dominada por un motivo central, cuyo propósito es realizar algo concreto y útil.

Las etapas del proyecto son [6]: 
1. Descubrimiento de una situación problemática, que necesita ser solucionada con la participación de todos los alumnos.

2. Definición y formulación del proyecto, diseñado por el profesor con la participación de todos los alumnos.

3. Planeamiento y complicación de datos, en la cual el profesor, por medio de preguntas y dudas aparentes, estimula a los alumnos para que elaboren el plan de trabajo y reflexiones acerca de las dificultades que encontrarán, y también dónde y cómo encontrar elementos para su ejecución.

4. Ejecución del proyecto. Los alumnos realizan las actividades propuestas en la planificación del proyecto, orientados, discretamente, por la acción del profesor.

5. Evaluación del proyecto, en la cual el profesor orienta el espíritu crítico de los alumnos acerca del proyecto en marcha o de sus resultados.

En conclusión, el método de proyecto se propone [7]:

a) Que los alumnos logren una situación auténtica de experiencia;

b) Que las actividades tengan propósitos definidos;

c) Que el pensamiento sea estimulado;

d) Que los resultados del trabajo sean algo concreto;

e) Que los alumnos tengan oportunidad de comprobar sus propias ideas a través de la aplicación de las mismas.

El origen de los trabajos didácticos orientados a los proyectos en la educación superior se inició al final de los años sesenta. Posteriormente, al comienzo de los setenta, se continuó con una etapa de mucha discusión y desarrollo práctico experimental. El sentido estricto de lo que se entiende como método de enseñanza orientada al proyecto, fue presentado a través de la definición elaborada por el British Council for National Academic Awards (CNAA), en 1972 [8], que dice: "Un proyecto es una investigación que satisface los requerimientos de las especificaciones aprobadas por el supervisor, éstas normalmente no tienen una sola respuesta correcta. Es la culminación de los estudios de un alumno de pregrado e implica la realización de juicios personales y la adopción de compromisos por parte de éste". En otras palabras, un proyecto es la búsqueda de una solución inteligente al planteamiento de un problema a resolver. En esta forma pueden haber diferentes ideas, inversiones, tecnologías y metodologías con diversos enfoques, todas ellas tendientes a resolver las necesidades del ser humano en todas sus facetas.
La educación organizada en proyectos es multidisciplinaria por naturaleza y puede ser dividida en dos grupos principales; puede orientarse al diseño o puede orientarse a la solución de los problemas. En el primer caso trata con los problemas prácticos construyendo y diseñando sobre la base de una síntesis de conocimientos de muchas disciplinas, en el segundo caso trata de la solución de los problemas teóricos a través del uso de cualquier conocimiento relevante, de cualquier disciplina de donde el conocimiento derive.

Para usar la metodología orientada al proyecto como elemento educativo central del plan de estudios, este tiene que ser organizado en "temas", escogidos de tal manera que la combinación de ellos logren plasmar el espíritu deseado en del plan de estudios y den forma al perfil profesional que se pretende obtener.

La metodología orientada al proyecto debe entenderse como un "sistema instruccional complementario", que atiende de mejor manera que el sistema tradicional, las necesidades del estudiante y del mercado. El énfasis en la cultura de trabajo en grupo, en primera instancia, produce un egresado más adaptado, y, por lo tanto, en mejores condiciones de ser contratado por las empresas. Opuestamente, los egresados de un sistema tradicional, con un proceso instruccional con más énfasis en el proceso de análisis, estarán mejor preparados en comprender los conceptos fundamentales y serán más capaces de trabajar independientemente; pero, requieren de mayor tiempo de entrenamiento en el lugar de empleo. Dicho de otra manera, la metodología orientada al proyecto pone más énfasis en los aspectos de eficiencia operacional e interpersonal, mientras que la educación tradicional se enfoca más bien hacia el logro de los conceptos fundamentales, de carácter teórico [9].

Uno de los desafíos más importantes que enfrenta en la actualidad la educación superior consiste en llegar a determinar anticipadamente las competencias profesionales que sus egresados necesitarán durante el transcurso de su ejercicio profesional. La metodología basada en los proyectos proporciona significativas respuestas a este desafío. Los proyectos conducen a un significativo nivel de práctica pre-profesional para los ingenieros en formación, proveyéndoles de las oportunidades de adquirir aquellas competencias que las empresas reiteradamente anhelan.

El sistema instruccional orientado al proyecto ha demostrado tener una gran capacidad de adaptación interna. No ha sido difícil ajustar y cambiar los programas educacionales de acuerdo con el desarrollo de la tecnología, la sociedad y la economía. El sistema es innovativo y ha sido capaz de lidiar con problemas usuales de la profesión y de la sociedad. Los resultados y la experiencia obtenida de la investigación que se 
lleva a cabo en las universidades es fácilmente incorporada en los programas de enseñanza, debido a la estrecha relación que existe entre este método con el proceso de solución de problemas [3].

Además, el sistema ha mostrado tener una gran capacidad de adaptación externa. Los egresados están bien preparados para resolver, en el trabajo futuro, problemas desconocidos y para extender su trabajo profesional más allá de lo esperado. Ellos también logran gran experiencia en equipos de trabajo interdisciplinarios y normalmente obtienen conocimientos de los últimos avances científicos y metodológicos. Algunas experiencias nos muestran resultados muy importantes que pueden ser logrados, utilizando el método de enseñanza orientada por proyecto. El desarrollo de la capacidad de integración social es quizás el más importante de estos resultados.

La forma de organización que se da a los alumnos en la metodología orientada al proyecto tiene el gran potencial de facilitar el aprendizaje, debido a la gran motivación que se logra entre los alumnos, involucrándose activamente en el proceso de aprendizaje y especialmente por sentirse atraídos por los relevantes aspectos de la vida real contenidos en los trabajos que se les asignan [10], [11].

\section{La Necesidad de Nuevos Modelos de Enseñanza}

Para nadie es un secreto en el sistema económico occidental que la forma tradicional de trabajo está obsoleta. Ha sido reemplazada por nuevos métodos integrativos. Esto incluye:

- Trabajo en equipo

- Gerenciamiento de calidad total

- Ingeniería sincrónica, que es mucho más que la ingeniería simultánea o concurrente; expresa la idea de trabajo en paralelo y también interconectividad intensiva

Los trabajadores, empresarios y los estudiantes tienen que prepararse, a través de su educación para estos nuevos métodos integrados de trabajo. Mientras mejor preparados, para estos aplicar nuevos conceptos mayores serán las posibilidades de éxito. Mientras más tecnología y organización necesite el hombre, mayor será la necesidad de nuevas formas de trabajo.

La educación tradicional se diseñó de acuerdo a las anteriores necesidades de las empresas:, los graduados son expertos en ciertas áreas temáticas, por ejemplo, ingenieros mecánicos, electrónicos y así sucesivamente. En sus cerebros se ha almacenado mucho conocimiento especializado, pero no se los ha dotado de la capacidad de buscar nuevos conocimientos. En contraste, la educación moderna debe enseñar el conocimiento necesario justo a tiempo y en el trabajo y desarrollar el aprender a aprender. En esta época, cuando el conocimiento crece rápidamente, el gerenciamiento del know-how es más necesario que nunca.

Consecuentemente, el enfoque de la ingeniería moderna aquí descrito enfatiza lo siguiente:

- La enseñanza del conocimiento básico

- Entrenamiento en el gerenciamiento del knowhow;

- La integración del hombre, la tecnología y la organización

La forma más eficiente de cumplir estas tres demandas es el trabajo orientado al proyecto durante el proceso de educación.

\section{La Formación Integral del Ingeniero y la Enseñanza Orientada al Proyecto}

Un informe de la UNESCO publicado por Delors en 1996, indica que la educación debe organizarse alrededor de cuatro tipos de aprendizaje, que serán la base de la vida de cada individuo [12]:

- Aprender a conocer, es decir, adquirir los instrumentos del conocimiento.

- Aprender a hacer, para poder actuar sobre el medio.

- Aprender a vivir juntos, para participar y cooperar con los demás en las actividades humanas.

- Aprender a ser, progresión esencial del ser humano que participa en los tres aprendizajes anteriores.

Estos cuatro pilares de la formación humana deberían tener el mismo tratamiento en una enseñanza estructurada, dado que la educación es para el individuo una experiencia global y continuada a lo largo de toda la vida. Más aún frente al paradigma de "formacióntrabajo", predominante hace unos años y caracterizado por la adquisición de un bagaje importante de conocimientos en las etapas iniciales de la vida, se ha pasado a un nuevo paradigma: La necesidad de una formación a lo largo de toda la vida.

El vertiginoso desarrollo de los conocimientos en general, y muy en particular en el campo de las tecnologías, así como su facilidad de acceso, hace que la formación inicial del ingeniero deba enfocarse sobre el desarrollo de capacidades intelectuales, no sólo como parte de su capacitación para el primer empleo, sino como base para mejorar su nivel de competencia y los posibles cambios de orientación de su vida activa. Esto 
conduce a lograr, entre otros, objetivos formativos como:

- Desarrollar el deseo de aprender, que potencie los hábitos de búsqueda del conocimiento en diversas fuentes

- Desarrollar una visión integradora, que posibilite el análisis de las dimensiones de cualquier problema o situación de la vida real, sin centrarse exclusivamente en la percepción parcial de una especialidad académica.

Estos principios son consecuentes con la metodología orientada al proyecto, pues promueve el trabajo en equipos multidisciplinarios con una visión integradora. Con todo, no debe olvidarse que el papel fundamental es la formación humana integral del individuo. En ese sentido debe evitarse el peligro de una formación desequilibrada, preocupada sólo por la capacitación del mercado laboral, y que olvide aspectos fundamentales de la persona. Por ello, de una visión de la educación puramente instrumental (habilidades, capacitaciones, fines económicos), debe llegarse a una concepción de la educación como una función global, que es la realización de la persona, el aprender ser. Esto nos lleva a tener presente que la formación humana debe ser un elemento fundamental en la educación en ingeniería de este nuevo milenio, como complemento a las dimensiones científicas, tecnológicas y económicas del perfil profesional del ingeniero. Hoy el mercado busca personas multifuncionales con actitudes básicamente humanas, como la capacidad de trabajar en equipo y con iniciativa. Esto conlleva el desarrollo de la "calidad humana" por encima de la "calidad profesional".

La globalización de la industria y del mercado laboral ha motivado la aparición de nuevas doctrinas laborales y educativas (economía global, interdependencia, el inglés como idioma universal de negocios, normalización internacional de productos, normas de calidad internacionales, cambio de valores en la empresa y en los individuos, fomento de la tecnología educativa, interacción entre las universidades, etc.). Todo ello lleva a definir nuevas líneas de desarrollo en la enseñanza de la ingeniería:

Desarrollar programas y normativas de enseñanza flexibles, capaces de adaptarse operacionalmente a los cambios del mercado laboral, línea que está en los preceptos de la enseñanza orientada al proyecto.

Desarrollar enseñanza humanística, incluyendo los idiomas extranjeros, la ética de la comunicación, las relaciones interpersonales, etc, como complemento imprescindible a la metodología propuesta.
Promocionar un sistema para mejorar la capacitación y la actualización del cuerpo docente en universidades y en empresas industriales, logrando así la integración deseada.

Estas acciones y otras que pueden sugerirse, deben de ser presididas por la idea de "convivencia", en donde se compartan recursos y conocimiento, y en donde el ingeniero pueda recibir una formación integral, para poder desarrollar los valores fundamentales de todo ser humano.

\section{Condiciones Requeridas}

La enseñanza orientada al proyecto requiere de un alto grado de supervisión por parte de los profesores. Además, contar con lugares adecuados de trabajos para los estudiantes. Cada grupo de proyecto necesita de un espacio en la universidad y, además, del proceso de supervisión continua de parte de un académico de la facultad. Las perspectivas siempre cambiantes, debidas a la adaptabilidad interna, requieren también de renovación de las clases o trabajo de aula. Esto supone mucha preparación y dedicación y puede afectar negativamente los recursos económicos de la institución. La adaptabilidad interna y la libre elección por parte de los estudiantes, crean una demanda de flexibilidad en los recursos de la universidad.

El contenido de los cursos debe ser integrado, introduciendo el estudio de casos o proyectos escogidos, de tal forma que calcen con los contenidos de varias materias, que permitan establecer situaciones en donde los estudiantes vayan desde la presentación de problemas complejos de la vida real, hasta la comprensión y manejo adecuado de definiciones y conceptos.

El rol de los profesores debe cambiar, puesto que ellos deben enfrentar requerimientos nacidos del sistema pedagógico propuesto. Estos requerimientos implican cambios en la metodología de la enseñanza y, en nuestro caso, también requiere de un periodo de entrenamiento en los aspectos básicos de la enseñanza orientada al proyecto. Ellos deben adoptar un nuevo papel y convertirse en facilitadores del aprendizaje, dando pocas respuestas y ofreciendo la oportunidad a los alumnos de hacer más preguntas. Por otra parte, la habilidad de los estudiantes para evaluar su propio desempeño debe ser mejorada, extendiendo su responsabilidad al incluir el establecimiento de estándares en los proyectos y así facilitar el proceso de auto evaluación de la ejecución de los mismos [5].

El sistema pedagógico en ingeniería debe ser desarrollado de manera tal, que asegure una relación dialéctica entre la teoría académica y la práctica 
profesional. Con esto se asegura una gran adaptabilidad de la aplicación de la teoría a la práctica y para la resolución de los problemas propuestos. Los problemas teóricos aseguran que este proceso pedagógico permite a los egresados mantenerse al día en sus conocimientos

Un sistema educacional exitoso depende de una interacción que comprenda profesión, investigación e instrucción. Los problemas que se encontrarán en la práctica profesional y en la investigación son las mejores guías para el proceso de aprendizaje. Ellos son los elementos que mantendrán a la práctica, la investigación y la educación unidos y que darán un resultado que será más fuerte que cada uno de sus componentes. Para lograr avances, debemos involucrar a la investigación y a la educación en el desarrollo del proceso, de tal manera que obtengamos una interacción dinámica [9].

\section{La Estructura Curricular de la Facultad de Ingeniería de la Universidad de Tarapacá}

La Facultad de Ingeniería de la Universidad de Tarapacá imparte las carreras de Ingeniería Civil e Ingeniería de Ejecución en las especialidades de: Electricidad, Electrónica, Industrias, Mecánica y Computación e Informática. La estructura de los planes de estudio es estratificada y de gran flexibilidad. Estas características permiten al alumno obtener Grados Académicos y Títulos Profesionales a medida que avanzan en la estructura curricular, Fig. 1 [10].

Esta estructura modular y secuencial sigue el siguiente esquema.

1) Bachillerato en Ingeniería: Con un plan de estudio común para todas las carreras de la Facultad, tanto Civil como de Ejecución. El Bachillerato tiene como objetivo proveer a los alumnos de una formación general, con una base de conocimientos adecuados que les permita continuar estudios hacía la Licenciatura o el Título Profesional. Este plan se compone de un conjunto de asignaturas que tienen un carácter esencialmente formativo en el campo de las ciencias básicas y de la ingeniería. Incluye, además, cursos electivos de formación humanística.

2) Ciclo de Especialidad: Con un plan común para ambos tipos de ingeniería y en cada especialidad. Las asignaturas de este ciclo mantienen el nivel y la orientación de Ingeniería de Ejecución. Incluye las Ciencias de Ingeniería propias de cada especialidad y en ellas se manifiesta la necesidad de una fuerte orientación hacia los aspectos específicos de aplicación. Al terminar este ciclo los alumnos deciden si continúan los estudios de Ingeniería de Ejecución o de Licenciatura en Ciencias de la Ingeniería, en la especialidad respectiva.

3) Etapa terminal de Ingeniería de Ejecución: Esta etapa está conformada principalmente por asignaturas de Formación Profesional de Especialidad, de acuerdo con la realidad tecnológica vigente. En el octavo semestre está ubicada la actividad de titulación para Ingenieros de Ejecución.

4) Etapa Licenciatura en Ingeniería: En esta etapa, ubicada en el octavo semestre, se obtiene el grado académico de Licenciado en Ciencias de la ingeniería y comprende los aspectos esenciales de un área de conocimiento o disciplina. En este período se incluyen asignaturas de Ciencias Básicas y Ciencias de Ingeniería, propias de cada especialidad. Sin embargo, a lo menos seis asignaturas son comunes a todas las especialidades. Al concluir estos estudios, los alumnos podrán optar por terminar el plan de Ingeniería Civil o realizar los estudios conducentes al grado de Magíster.

5) Etapa terminal de Ingeniería Civil: En ella se considera un conjunto de asignaturas de formación profesional y formación general, que definen el perfil correspondiente a cada especialidad de Ingeniería Civil. En los semestres undécimo y duodécimo está ubicada la actividad de titulación. 


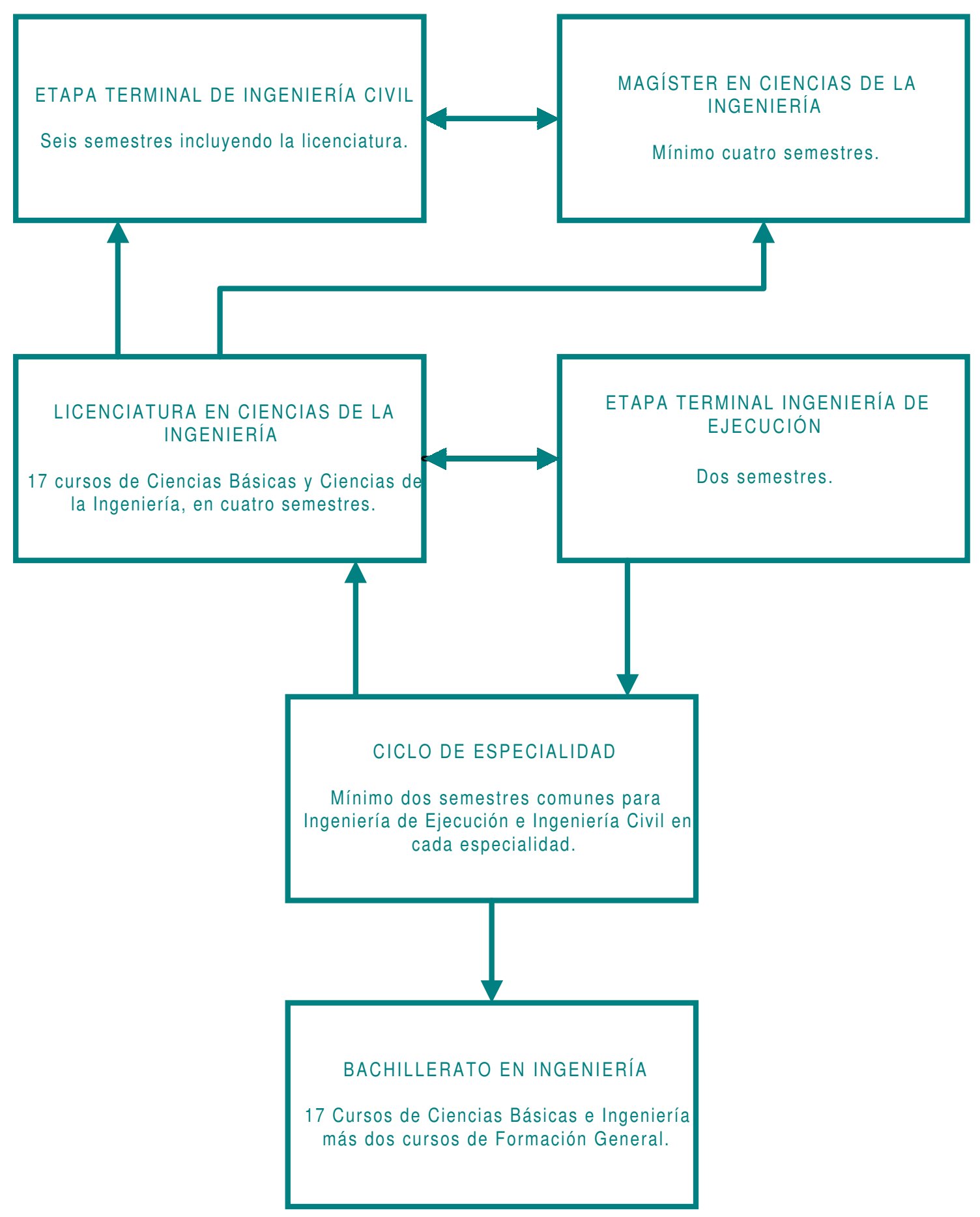

Fig. 1 Estructura General de los planes de estudio por especialidad, de la Facultad de Ingeniería. 

6) Etapa terminal de Ingeniería de Ejecución: Esta etapa está conformada principalmente por asignaturas de Formación Profesional de Especialidad, de acuerdo con la realidad tecnológica vigente. En el octavo semestre está ubicada la actividad de titulación para Ingenieros de Ejecución.

7) Etapa Licenciatura en Ingeniería: En esta etapa, ubicada en el octavo semestre, se obtiene el grado académico de Licenciado en Ciencias de la ingeniería y comprende los aspectos esenciales de un área de conocimiento o disciplina. En este período se incluyen asignaturas de Ciencias Básicas y Ciencias de Ingeniería, propias de cada especialidad. Sin embargo, a lo menos seis asignaturas son comunes a todas las especialidades. Al concluir estos estudios, los alumnos podrán optar por terminar el plan de Ingeniería Civil o realizar los estudios conducentes al grado de Magíster.

8) Etapa terminal de Ingeniería Civil: En ella se considera un conjunto de asignaturas de formación profesional y formación general, que definen el perfil correspondiente a cada especialidad de Ingeniería Civil. En los semestres undécimo y duodécimo está ubicada la actividad de titulación.

\section{Análisis del Curriculum Desde la Perspectiva de la Enseñanza Orientada al Proyecto}

La pregunta curricular que surge al diseñar la instrucción es la siguiente: ¿qué deben enseñar los profesores?. La labor de los profesores consiste fundamentalmente en preparar a las nuevas generaciones, enseñándoles los últimos conocimientos, que permitan a los estudiantes mantenerse vigentes en su profesión. Sin embargo, dado el rápido desarrollo del conocimiento ¿cómo se puede estar seguro de ello?. Como respuesta a esta última pregunta, el trabajo orientado a proyectos en sus conceptos manifiesta lo siguiente: "Es el intento de resolver los problemas actuales de la ingeniería lo que guía a los estudiantes junto con los profesores a encontrar el conocimiento y las teorías que son esenciales".

El sistema instruccional debe ser diseñado de tal manera que asegure una relación dialéctica entre la teoría académica y la práctica profesional. Esto permitirá una gran adaptabilidad de aplicación entre teoría y práctica, con el propósito de resolver problemas. Los problemas teóricos potencian la maquinaria que opera el proceso instruccional y asegura que el conocimiento de nuestros egresados esté al día.
Insistimos, el sistema educacional exitoso depende de una interacción entre profesión, investigación y educación. Los problemas que se encuentran en la práctica profesional y la investigación son la mejor guía para el proceso de aprendizaje y son los elementos que dan coherencia al sistema.

La práctica puede ser definida como los campos específicos o tareas que conforman funciones profesionales dentro de la sociedad y éstas son llevadas a cabo por personas académicamente entrenadas, en nuestro caso, por los ingenieros.

En la sociedad actual de creciente complejidad, se deben enfrentar continuamente nuevos problemas y nuevos desafíos. El desarrollo tecnológico, la manufactura computacional integrada y los sistemas expertos son ejemplos de esta demanda dentro de nuestros campos profesionales o dentro de nuestra práctica. Los caminos tradicionales para enfrentar estos desafíos son el entrenamiento en el lugar de trabajo, seminarios profesionales, publicaciones de artículos, etc. Estos métodos de desarrollo son procesos lentos.

Las respuestas e incluso los problemas en sí pueden ser no relevantes cuando se encuentran las soluciones, mientras la sociedad continúa enfrentando nuevos problemas que requieran nuevas soluciones

Debemos enfrentar el hecho de que los métodos tradicionales no son siempre los más adecuados. Las respuestas no están siempre dentro de las mismas profesiones, sino en el trabajo muldisciplinario. Para lograr ser mejores debemos involucrar la investigación y ésta en el proceso de desarrollo, de tal manera que obtengamos un intercambio dinámico.

Necesitamos investigar para producir respuestas teóricas, y necesitamos interactuar con la educación para producir profesionales capaces de dar respuestas prácticas, mediante la aplicación de nuevos conocimientos y habilidades, para enfrentar los nuevos y aún desconocidos problemas del futuro.

En nuestro caso, el nivel de Bachiller en Ingeniería es impartido para estudiantes de todas las especialidades dictadas en la Facultad de Ingeniería, en cursos que son regularmente demasiados numerosos. La mayoría de estos cursos son de Ciencias Básicas y algunos de Ciencias de la Ingeniería, sin ningún tipo de integración entre ellos y en un esquema donde los estudiantes difícilmente visualizan la aplicación del conocimiento que reciben. El grado de dificultad y los costos para una reforma aparentemente son muy altos, sin embargo, hay algunas medidas que se podrían adoptar a partir del tercer semestre de la carrera, entre otras: 
- Integrar materias tales como Ecuaciones Diferenciales I, que pretende lograr al final del curso que el alumno sea capaz de describir, analizar, resolver e interpretar las Ecuaciones Diferenciales ordinarias asociadas a sistemas físicos y Computación II que pretende lograr al final del curso que el alumno sea capaz de describir los algoritmos de solución de los problemas a través de programas escritos en un lenguaje de programación moderno, como también mediante software computacional disponible en forma de paquetes. En fin, se podría plantear numerosas posibilidades de integración, que permitan al alumno trabajar en proyectos asociados a problemas reales. O sea, diseñar proyectos que hagan más interesante $\mathrm{y}$ motivador el estudio y el aprendizaje en un nivel en donde los porcentajes de deserción son elevados, haciendo aparecer el sistema como poco eficiente.

- Es posible estudiar diversas reformas en este sentido, quitándole aridez al aprendizaje de las ciencias básicas y haciendo más interesante el aprendizaje de la enseñanza de la Electrotecnia y la Computación, por ejemplo. Aún más, algunos conceptos de Ciencias Básicas podrían ser asimilados a asignaturas de Ciencias de la Ingeniería, como por ejemplo Física del Electromagnetismo e Ingeniería Electromagnética, integrándose a proyectos que permitan al alumno entender la necesidad de adquirir tal conocimiento.

- Las otras etapas de la estructura del curriculum son principalmente de Ciencias Aplicadas, con excepción del nivel denominado Licenciatura en Ciencias de la Ingeniería. Las Ciencias Aplicadas tienen que ver con problemas que surgen de la práctica. Es característico, por la naturaleza de los problemas de las Ciencias Aplicadas, que estos estén directamente relacionados con aquellos que los ingenieros enfrentarán en la vida real, los cuales están lejos del mundo de las Ciencias Puras. Es en el intercambio dialéctico entre Ciencias puras y Ciencias Aplicadas que se encuentra el progreso de la ciencia. Las Ciencias Aplicadas impulsan a las Ciencias Básicas a progresar en aquellas áreas donde los problemas prácticos aparecen o emergen con más frecuencia Como resultado, las Ciencias Aplicadas se desarrollan sobre la base de nuevas teorías y metodologías, que les proporcionan las Ciencias Puras.

- Las Ciencias Aplicadas son por esencia "orientadas al proyecto". La Educación orientada al proyecto es multidisciplinaria por esencia. Puede dividirse en dos grandes grupos: "orientada al diseño" y " orientada a los problemas de instrucción". Los proyectos orientados al diseño tratan con la solución de problemas prácticos, construyendo y diseñando sobre síntesis del conocimiento de varias disciplinas. Los proyectos orientados a los problemas de instrucción tratan con la solución de problemas teóricos a través del uso de los conocimientos relevantes, tomados desde cualquier disciplina de donde ellos provengan. En los programas de ingeniería podremos usar ambas clases de proyectos

- $\quad$ Por la naturaleza del Ciclo de Especialidad y de la Etapa terminal de Ingeniería de Ejecución parece lógico dar énfasis principalmente al método orientado al diseño, dado que este método de enseñanza permitiría dar un enfoque multidisciplinario a las asignaturas de cada especialidad de la ingeniería e incluso realizar proyectos llevados a cabo por ingenieros de distintas especialidades. Aún más, la aplicación de la enseñanza orientada al proyecto en el Ciclo de Especialidad permitiría a los futuros alumnos de la Licenciatura en Ciencias de la Ingeniería, una cabal comprensión de las posibilidades de aplicación de las asignaturas de Ciencias Básicas Avanzadas y de Ingeniería que allí se estudiarán.

- Tal como se mencionó anteriormente en los programas de ingeniería, podría aplicarse ambos tipos de educación orientada al proyecto, en la etapa terminal de Ingeniería de Ejecución el trabajo de proyecto debería estar más orientado a la metodología, al diseño, mientras que la Ingeniería Civil debería involucrar principalmente la metodología orientada a los problemas, esto debido a la diferente naturaleza de los planes de estudio y basándose en los conceptos expuestos con anterioridad.

\section{CONCLUSIONES}

Una de las estrategias de docencia, incorporada en el plan de desarrollo de la Facultad de Ingeniería de la Universidad de Tarapacá, para la optimización continua de los recursos y la determinación de nuevas alternativas y tendencias, puede ser el método de proyectos. Entre los más importantes desafíos que enfrenta la educación en ingeniería está lograr anticipar las competencias que nuestros egresados necesitarán poseer durante el desarrollo de su profesión Para el cumplir este desafío se requiere que la educación en ingeniería responda a las actuales y futuras necesidades de nuestra sociedad. La educación basada en proyectos da una significativa respuesta a estos desafíos. Los proyectos conducen a un importante nivel de práctica pre-profesional para los ingenieros en formación, dando a los estudiantes importantes oportunidades de aprender 
aquellas habilidades que nuestra sociedad e industrias solicitan.

Indudablemente, la implantación de esta metodología implica una importante inversión en estructura, capacitación de los docentes y, principalmente, un significativo trabajo en la modificación de los programas y metodología de la enseñanza de la ingeniería. No parece muy lejana la posibilidad de aplicar este sistema de enseñanza a los niveles correspondientes a los ciclos de Especialidad y etapas terminales de Ingeniería de Ejecución e Ingeniería Civil, dado que por su naturaleza parecen destinadas a emplear el método de enseñanza de la ingeniería orientada al proyecto, con todas sus virtudes de integración multidisciplinaria y de gran participación del estudiante en el proceso de enseñanza-aprendizaje.

\section{REFERENCIAS}

[1] Klaus Peschges, Erich Reindel; 'Project-Oriented Engineering Education to Improve Key Competencies", Global J. of Engineering. Educ., Vol. 2, N², 1998.

[2] Stig Enemark, "Project -Organized Education The Basic Principle", Workshop for ITESM Academic Directors, Aalborg University Denmark, 2003.

[3] The Aalborg Experiment Project Innovation in University Education. http://www.teknat.auc.dk

[4] I.G. Nérici; 'Hacia una didáctica general dinámica”, editorial Kapelusz, Buenos Aires, 1985.
[5] A. Valcárcel; 'Didáctica universitaria”, editorial La Muralla, S.A., Madrid, 2001.

[6] C. Monereo y otros; 'Estrategias de enseñanza y aprendizaje", editorial Grao, Barcelona, 1997.

[7] A. Zabala; 'La práctica educativa. Cómo enseñar", editorial Grao, Barcelona, 2000.

[8] British Council for National Academic Awards (CNAA), 1972.

[9] Project Organised Education The Role of the Students. http://www.ale2002.dtv.dk

[10] Lance Schachterler, Ole Vinther; "Introduction The Role of Projects in Engineering Education", European Journal of Engineering Education, Vol. 21, $\mathrm{N}^{\circ} 21, \mathrm{~N}^{\circ} 2,1996$.

[11] Klaus Peschges, Erich Reindel; "How to Structure and Mark Project-Oriented Studies", Global J. of Engineering Educ., Vol. 3, N³, 1999.

[12] Jacques Delors, Informe UNESCO, 1996.

[13] Knud Nielsen, Ole Vinther, "Experiences of Success and Failure in Project Organization", European Journal of Engineering Education Vol. 2, 1996.

[14] Facultad de Ingeniería, "Planes de Estudio", Universidad de Tarapacá, 1995. 